\title{
Infrastructure maxillectomy for maxillary sinus and hard palate neoplasms
}

\author{
DONG HOON LEE, HYE RIN LIM, JOON KYOO LEE and SANG CHUL LIM
}

\begin{abstract}
Department of Otolaryngology-Head and Neck Surgery, Chonnam National University Medical School and Chonnam National University Hwasun Hospital, Hwasun, Jeonnam 58128, Republic of Korea
\end{abstract}

Received November 22, 2020; Accepted June 1, 2021

DOI: $10.3892 / \operatorname{mco} .2021 .2342$

\begin{abstract}
Infrastructure maxillectomy is a surgical procedure to remove the lower part of the maxilla and hard palate. The objective of the present study was to analyze clinical data and treatment outcome of patients who underwent infrastructure maxillectomy between 2011 and 2019. A total of 13 patients who underwent infrastructure maxillectomy for maxillary sinus and hard palate neoplasms between 2011 and 2019 were analyzed. These patients were subdivided into maxillary sinus neoplasm $(n=5)$ and hard palate neoplasm $(n=8)$ groups. All patients except one underwent infrastructure maxillectomy using the sublabial approach. One patient underwent an external approach through lateral rhinotomy. Postoperative reconstruction was performed for 11 patients using obturator, 6 patients using skin grafts and 3 patients using free flaps. A total of 6 patients had radiotherapy (RT), 3 had concurrent chemoradiotherapy (CCRT) and 2 had chemotherapy after surgery. The survival rate and recurrence rate were $61.5 \%(8 / 13)$ and $46.2 \%(6 / 13)$, respectively. The current results suggested that infrastructure maxillectomy may be an effective treatment for maxillary sinus neoplasms in the lower part of the maxillary sinus and hard palate neoplasms without causing marked functional or cosmetic morbidity. Postoperative RT or CCRT may be recommended to decrease the recurrence after infrastructure maxillectomy.
\end{abstract}

\section{Introduction}

Infrastructure maxillectomy is a surgical procedure to remove the lower part of the maxilla and hard palate $(1,2)$. The

Correspondence to: Professor Sang Chul Lim, Department of Otolaryngology-Head and Neck Surgery, Chonnam National University Medical School and Chonnam National University Hwasun Hospital, 322 Seoyang-ro, Hwasun, Jeonnam 58128, Republic of Korea

E-mail: limsc@jnu.ac.kr

Key words: maxillary neoplasms, hard palate, malignant, oral surgical procedures, radiotherapy procedure preserves structures around orbit and zygoma, thus providing good functional and cosmetic results (3). However, the role of partial maxillectomy for maxillary sinus and hard palate carcinomas is not well understood yet (2). Therefore, the objective of this study was to analyze clinical data and treatment outcome of patients who underwent infrastructure maxillectomy between 2011 and 2019.

\section{Patients and methods}

This study was approved by the Institutional Review Board of Chonnam National University Hwasun Hospital (CNUHH-2020-049). Sixteen patients who underwent infrastructure maxillectomy for maxillary sinus and hard palate neoplasms between 2011 and 2019 were identified. All patients provided written informed consent. Among them, 3 patients who had reoperation for maxillary sinus cancer were not analyzed. These subjects were subdivided into maxillary sinus neoplasm $(n=5)$ and hard palate neoplasm $(n=8)$ groups. We reviewed their demographic characteristics, symptoms, duration of symptoms, size and location of primary tumor, preoperative biopsy results, radiologic examinations, clinical stage, surgery, reconstruction method, histopathologic results, treatment outcomes, adjuvant treatment, postoperative complications, and recurrence.

All patients underwent radiologic examinations before the operation to assess the extent of the lesion and aid treatment planning. If lymph node metastasis was suspected or diagnosed by preoperative examinations, a neck dissection was performed. All patients who underwent infrastructure maxillectomy were confirmed histopathologically. RT or CCRT was performed according to postoperative biopsy results.

\section{Results}

Of 13 patients who underwent infrastructure maxillectomy, the location of the primary tumor was maxillary sinus in 5 patients and hard palate in 8 patients. Clinical findings of patients who underwent infrastructure maxillectomy are summarized in Table I. There were 11 males and 2 females. The mean age of all patients was $67.4 \pm 10.5$ years (range, $43-80$ years). The most common symptoms were pain $(n=6,46.2 \%)$, abnormal sensation $(n=2)$, rhinorrhea $(n=2)$, mass $(n=1)$, cheek swelling $(n=1)$, and oral bleeding $(n=1)$. 


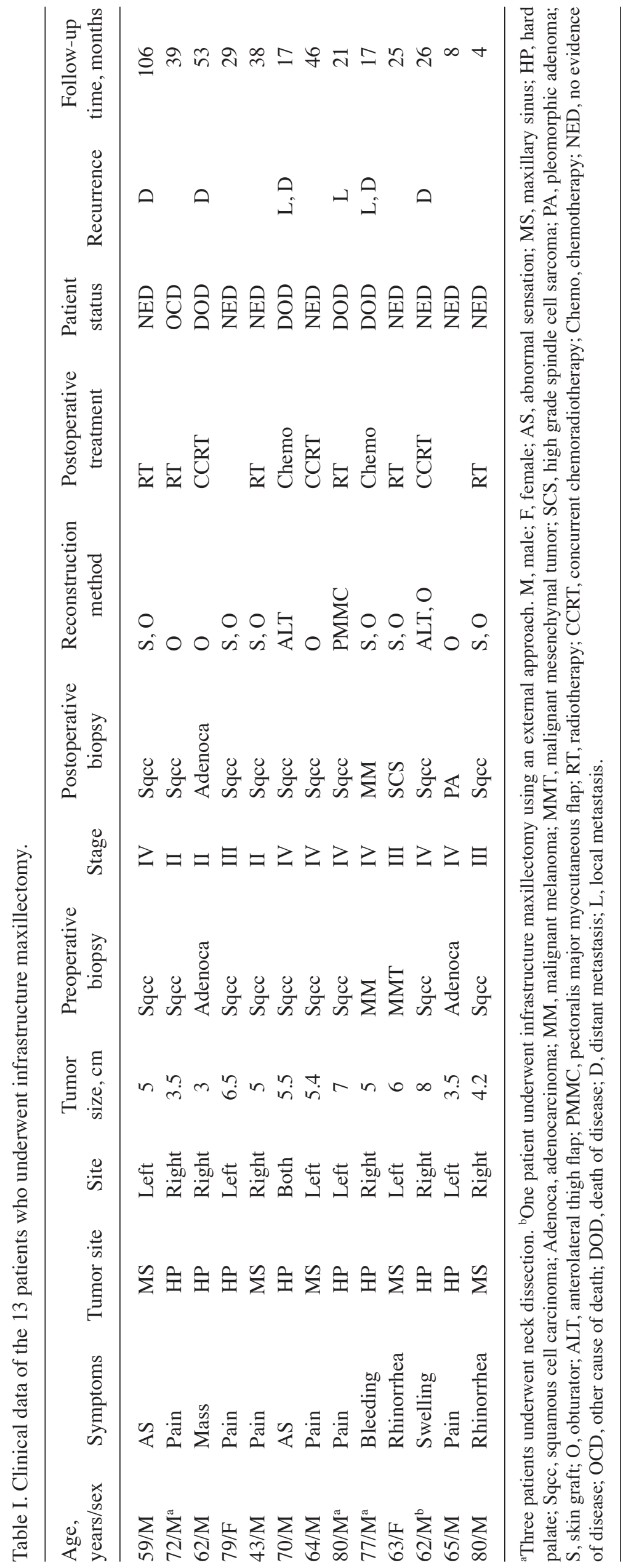


A
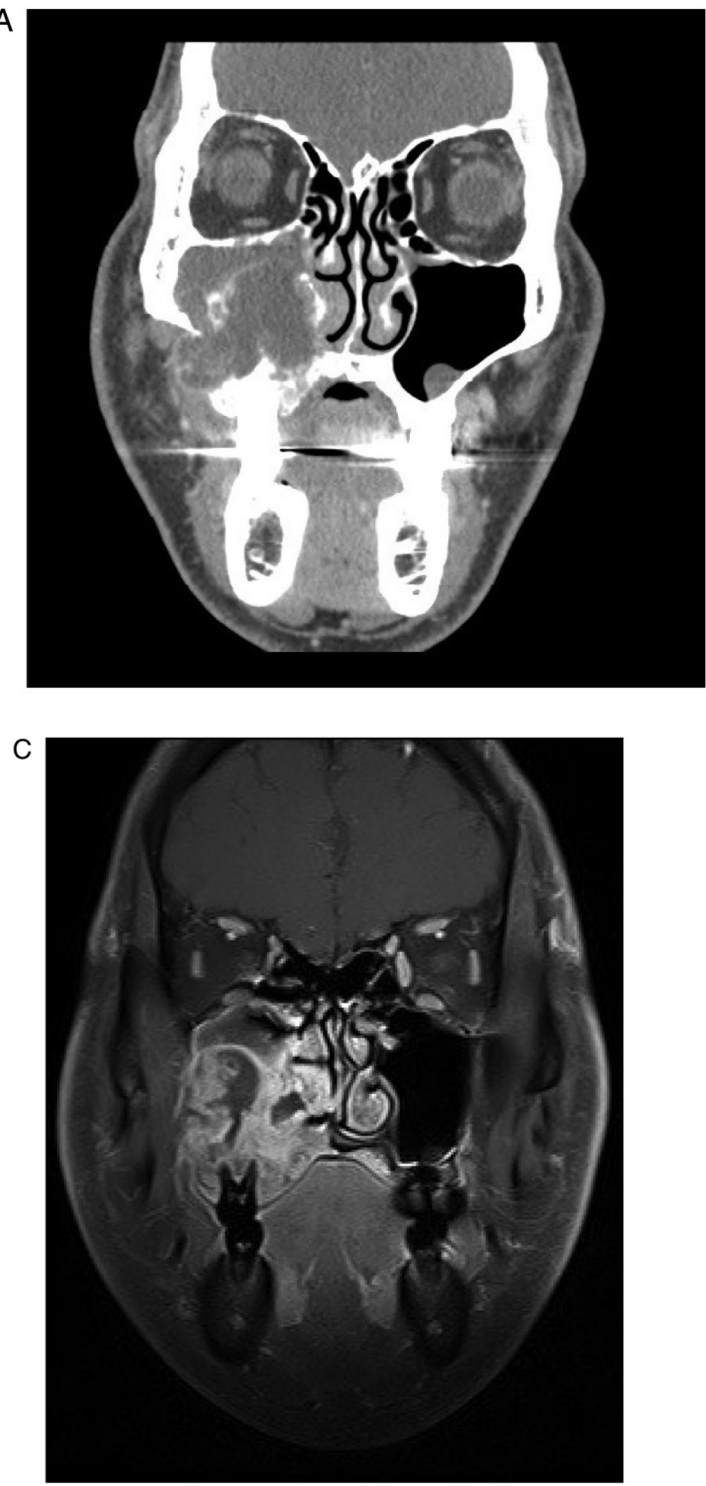

B

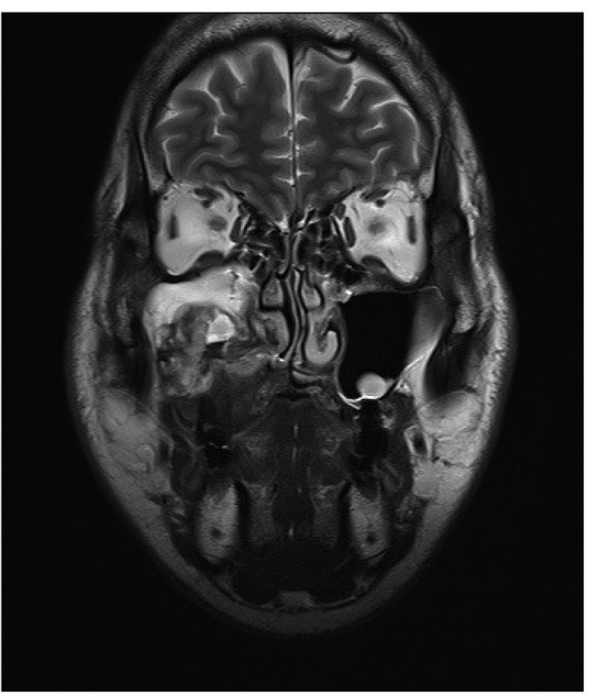

D

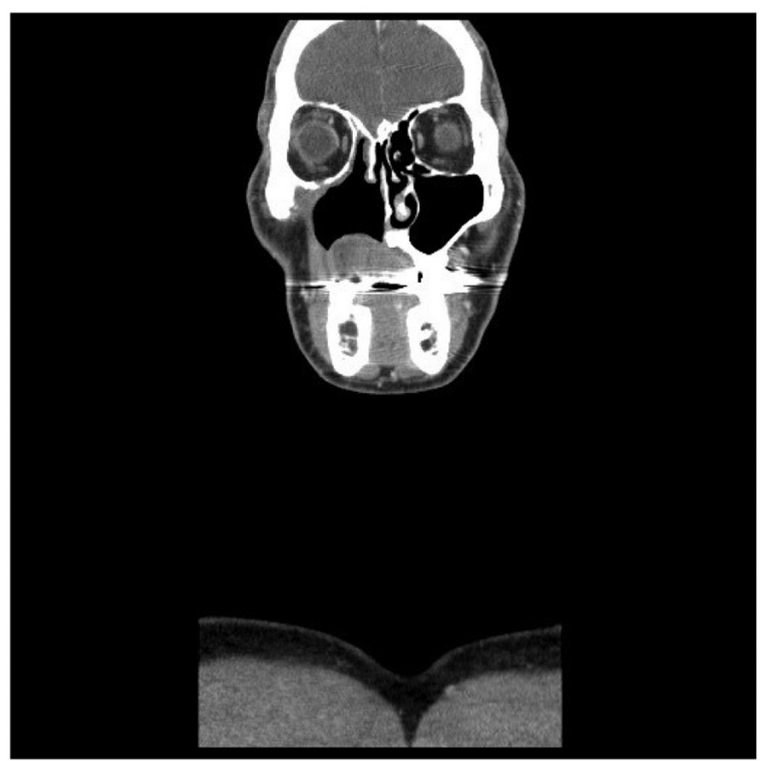

Figure 1. In a 43-year-old male patient, (A) preoperative coronal CT, (B) coronal T2-weighted MRI and (C) coronal T1-weighted MRI with enhancement showing $5 \times 4.6 \mathrm{~cm}$ sized multi-loculated cystic mass in the right maxillary sinus. (D) Coronal CT showing no recurrence at three years after treatment with infrastructure maxillectomy and postoperative radiotherapy. CT, computed tomography; MRI, magnetic resonance imaging.

The mean duration of symptoms was $1.8 \pm 1.5$ months (range, 0.2-6 months). Of 13 patients, 6 occurred on the left side, 6 on the right side, and 1 on both sides. The mean tumor size was $5.2 \pm 1.5 \mathrm{~cm}$ (range, $3-8 \mathrm{~cm}$ ).

Preoperative punch biopsy was performed for all patients. Results revealed that squamous cell carcinoma was the most common in 9 patients $(n=9,69.2 \%)$, followed by adenocarcinoma $(n=2)$, malignant melanoma $(n=1)$, and malignant mesenchymal tumor $(n=1)$. Computed tomography $(\mathrm{CT}, \mathrm{n}=10)$, magnetic resonance imaging $(\mathrm{MRI}, \mathrm{n}=5)$, and positron emission tomography CT (PET CT, $n=10)$ were performed to confirm primary site lesions, neck and distant metastasis. Before surgery, 2 patients underwent chemotherapy and 1 underwent chemotherapy and CCRT. In the clinical stage, stage IV was the most common $(n=7,53.8 \%)$. Stage III and stage II had 3 patients each. There was no patient with stage I.

All patients except one underwent infrastructure maxillectomy using the sublabial approach. One patient underwent an external approach through lateral rhinotomy. We performed frozen biopsies to determine whether further resection was needed. Resection of lesions with clear margins was performed. Neck dissection was performed in 3 patients. Postoperative reconstruction was done for 11 patients using obturator, 6 with skin graft, and the 3 with free flaps [ 2 with anterolateral thigh (ALT) flap and 1 with pectoralis major myocutaneous (PMMC) flap]. No major complications from surgical intervention were reported. Histopathologic results included squamous cell carcinoma $(n=9$, Fig. 1$)$, adenocarcinoma $(n=1)$, malignant melanoma $(n=1)$, high grade spindle cell sarcoma $(n=1)$, and pleomorphic adenoma $(n=1)$. Histopathologic examination revealed that one patient who was considered as having adenocarcinoma on preoperative biopsy was found to have pleomorphic adenoma. Except for this one, the final biopsy was the same as the preoperative biopsy. Among 13 patients who underwent infrastructure maxillectomy, 6 had RT, 3 had CCRT, and 2 had chemotherapy after surgery. One patient diagnosed with squamous cell carcinoma (stage III) of the hard 
palate and one patient with pleomorphic adenoma as a final diagnosis did not undergo additional treatment after surgery.

Among 13 patients who underwent infrastructure maxillectomy, 5 patients (38.5\%) died during the follow-up, including 4 tumor related deaths and 1 death from other disease. Recurrence occurred in 6 (1 case of local recurrence, 3 cases of distant metastasis, and 2 cases of local and distant recurrence). Sites of distant metastasis were lung in 3 patients, mediastinum in 1 patient, and whole body in 1 patient diagnosed with malignant melanoma. Three of five patients who developed distant metastases died and two were followed up without recurrence after lung metastasis resection and treatment. The mean follow-up period after surgery was

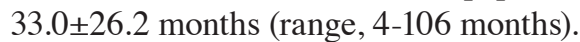

\section{Discussion}

This study presented our 9-year experience of infrastructure maxillectomy for maxillary sinus and hard palate neoplasms. The survival rate of patients who underwent infrastructure maxillectomy at the last follow-up was $61.5 \%$ (8/13), similar to previous reports, although disease location, stage, and histology were different between our study and previous studies $(2,4)$.

We performed infrastructure maxillectomy for 5 maxillary sinus neoplasms and 8 hard palate neoplasms. Imaging examinations such as CT and MRI can confirm the exact extent of the lesion. Infrastructure maxillectomy can provide sufficient oncological safety margin (2). In addition, it can determine the clear resection margin through frozen biopsies (2). In all cases, we confirmed clear surgical margins by intraoperative frozen biopsies.

Preoperative punch biopsy was done for all patients. Result of preoperative punch biopsy was the same as the final biopsy after surgery except for 1 patient. Therefore, it is better to perform a preoperative punch biopsy to determine the surgical plan such as metastasis evaluation and reconstruction method.

All patients except one were operated via sublabial approach without external incision. In one case, the hard palate carcinoma invaded the nasal alar and surrounding tissues. Thus, external approach through lateral osteotomy was inevitable. For 3 patients, neck dissection was performed due to the presence of preoperatively suspicious metastatic lymph node. In 11 patients, postoperative RT, CCRT, or chemotherapy was required to treat residual microscopic disease to achieve more satisfactory local control and reduce the rate of recurrence $(5,6)$. Indications of postoperative RT or CCRT include advanced stage, positive or close surgical margins, perineural invasion, and neck lymph node metastasis (6). Two patients (1 patient with malignant melanoma and 1 patient who received CCRT before surgery) received chemotherapy only.

After infrastructure maxillectomy, a palatal defect will develop. It must be sealed (1,7-9). The method of palatal defect reconstruction depends on the size of the defect and the availability of dentition to support prosthesis (1). The reconstruction method for a palatal defect includes an obturator, a locoregional pedicled flap, or a free flap (1,7-9). The most common reconstruction method in this study was obturator $(84.6 \%, 11 / 13)$. Free flap has the advantage of being able to supply virtually unlimited tissue. However, it has increased surgical time and donor site morbidity (9). Free flap was performed only in 3 patients with large and wide lesions in the present study.

Survival rate and recurrence rate were $61.5 \%$ (8/13) and $46.2 \%$ (6/13), respectively. These results were similar to those of total or radical maxillectomy $(2,3,10)$. Postoperative RT or CCRT is needed in most cases, even with partial or total maxillectomy (2). In addition, infrastructure maxillectomy can remarkable reduce functional and cosmetic damage due to preservation of orbit and zygoma $(2,3)$. Therefore, this surgical method is an effective treatment for maxillary sinus neoplasms in the lower part of the maxillary sinus and hard palate neoplasms.

The limitation of this study is the small sample size. Long-term follow-up of more patients at multiple centers is required. Another limitation of this study is the absence of figures of before and after surgery or histopathological findings.

Infrastructure maxillectomy is an effective treatment for maxillary sinus neoplasms in the lower part of the maxillary sinus and hard palate neoplasms without causing remarkable functional or cosmetic morbidity. We recommend postoperative RT or CCRT to reduce recurrence after an infrastructure maxillectomy.

\section{Acknowledgements}

Not applicable.

\section{Funding}

No funding was received.

\section{Availability of data and materials}

All data generated or analyzed during this study are included in this published article.

\section{Authors' contributions}

DHL analyzed and interpreted the patient data regarding the disease, and was a major contributor in writing the manuscript. DHL, JKL and SCL performed the infrastructure maxillectomy. DHL and SCL conceived and designed the study. DHL, HRL and SCL acquired the data. DHL and HRL analyzed and interpreted the data. All authors confirm the authenticity of all the raw data and have read and approved the final manuscript.

\section{Ethics approval and consent to participate}

The study was approved by the Institutional Review Board of Chonnam National University Hwasun Hospital (approval no. CNUHH-2020-049). All patients provided written informed consent.

\section{Patient consent for publication}

Written informed consent was obtained for the publication of patient images. 


\section{Competing interests}

The authors declare that they have no competing interests.

\section{References}

1. Omura K, Nomura K, Aoki S, Otori N and Tanaka Y: Soft tissue reconstruction with anterior pedicled inferior turbinate flap in conjunction with palatal flap for standard inferior maxillectomy with hard palate resection. Head Neck 42: 1110-1114, 2020.

2. Roy BC, Bahadur S and Thakar A: Partial maxillectomy for malignant neoplasms of para nasal sinuses and hard palate. Indian J Cancer 39: 83-90, 2002.

3. Liu L, Liu D, Guo Q and Shen B: Quality of life in advanced maxillary sinus cancer after radical versus conservative maxillectomy. J Craniofac Surg 24: 1368-1372, 2013.

4. Truitt TO, Gleich LL, Huntress GP and Gluckman JL: Surgical management of hard palate malignancies. Otolaryngol Head Neck Surg 121: 548-552, 1999.
5. Aydil U, Kizıl Y, Bakkal FK, Köybasıŏlu A and Uslu S: Neoplasms of the hard palate. J Oral Maxillofac Surg 72: 619-626, 2014

6. Li Q, Zhang XR, Liu XK, Liu ZM, Liu WW, Li H and Guo ZM: Long-term treatment outcome of minor salivary gland carcinoma of the hard palate. Oral Oncol 48: 456-462, 2012.

7. King E, Abbott C, Dovgalski L and Owens J: Orofacial rehabilitation with zygomatic implants: CAD-CAM bar and magnets for patients with nasal cancer after rhinectomy and partial maxillectomy. J Prosthet Dent 117: 806-810, 2017.

8. Murphy J, Isaiah A, Wolf JS and Lubek JE: Quality of life factors and survival after total or extended maxillectomy for sinonasal malignancies. J Oral Maxillofac Surg 73: 759-763, 2015.

9. Freije JE, Campbell BH, Yousif NJ and Matloub HS Reconstruction after infrastructure maxillectomy using dual free flaps. Laryngoscope 107: 694-697, 1997.

10. Hayashi T, Nonaka S, Bandoh N, Kobayashi Y, Imada M and Harabuchi Y: Treatment outcome of maxillary sinus squamous cell carcinoma. Cancer 92: 1495-1503, 2001. 